\title{
Wind of presupernova IIn SN 1997eg
}

\author{
N.N. Chugai \\ Institute of astronomy RAS, Moscow 119017, Pyatnitskaya 48 \\ nchugai@inasan.ru
}

\begin{abstract}
Spectra and phototometry of type IIn supernova SN 1997eg are used to determine properties of the circumstellar gas lost by the presupernova during the latest 200 years before the explosion. The analysis of narrow $\mathrm{H} \alpha$ and [Fe X] $6374 \AA$ results in the wind velocity $u=20 \mathrm{~km} \mathrm{~s}^{-1}$, significantly lower than the earlier accepted value $\left(160 \mathrm{~km} \mathrm{~s}^{-1}\right)$ upon the bases of the radial velocity of a blue absorption wing of the narrow $\mathrm{H} \alpha$. That high velocity of the wind in our picture is related to the preshock gas accelerated by the cosmic ray precursor. The modelling of the circumstellar interaction results in the wind density parameter $\dot{M} / u$ that being combined with the wind velocity suggests the presupernova mass loss rate of $1.6 \cdot 10^{-3} M_{\odot} \mathrm{yr}^{-1}$. The wind density is consistent with the [Fe X] $6374 \AA$ luminosity. The model $\mathrm{H} \alpha$ luminosity also agrees with the observational value. Recovered wind properties indicate that the presupernova at the final evolutionary stage was a massive red supergiant with a high mass loss rate, but not the LBV-supergiant as suggested earlier.
\end{abstract}

\section{Introduction}

Supernova SN 1997eg (Nakano \& Aoki 1997) belongs to type IIn (Filippenko \& Barth 1997) with signatures of a dense circumstellar matter (CSM). The luminosity of most SNe IIn both in lines and continua is due to the dissipation of a kinetic energy in forward and reverse shocks in the course of the SN/CSM interaction. Likewise in the canonical SN $1988 Z$ (Filippenko 1991, Statakis \& Sadler 1991), strong emission lines of SN 1997eg show three components (Hoffman et al. 2008): the narrow lines of the CSM, the broad component $\left(\mathrm{FWHM} \approx 10^{4} \mathrm{~km} \mathrm{~s}^{-1}\right)$ and the intermediate component $\left(\mathrm{FWHM} \approx 2000 \mathrm{~km} \mathrm{~s}^{-1}\right)$. The broad component originates from the undisturbed SN envelope and the cool dense shell (CDS) between forward and reverse shocks. The intermediate component originates from dense CS clouds interacting with the forward shock (Chugai \& Danziger 1994).

The origin of SNe IIn, particularly, the main-sequence mass and mass loss mechanism, are far from clear. In this regard of great importance are properties of the CSM, i.e., the 
velocity, density and spatial scale of CSM distribution, which could shed light on the issue. It is noteworthy, the stellar wind is not the only potential source of the dense CSM. In some cases the CSM can be a result of a mass ejection in a single explosive perturbation likewise in SN 2006gy (Woosley et al. 2007). Insight into the CSM density can be obtained via the modelling of the bolometric luminosity and the evolution of the supernova expansion velocity. The later is recovered from emission line profiles. As to the bolometric luminosity of SN 1997eg the situation is hampered by the absence of long term observations. Yet several photometric observations around day 90 (Tsvetkov \& Pavlyuk 2004) in combination with estimates of the supernova velocity could be sufficient for the determination of the CSM density.

With the known density and wind velocity one immediately recovers the mass loss rate. In most SN IIn however the low spectral resolution does not permit to infer the wind velocity. In this regard of particular significance is the spectrum of SN 1997 eg on 198 day with the resolution of $7 \mathrm{~km} \mathrm{~s}^{-1}$ (Salamanka et al. 2002). Yet the former interpretation of narrow lines is doubtful. The point is that apart from narrow emission lines with FWHM of $30-40 \mathrm{~km} \mathrm{~s}^{-1}$ the spectrum shows broad absorption component of $\mathrm{H} \alpha$ with the radial velocity $-160 \mathrm{~km} \mathrm{~s}^{-1}$ in the blue wing, which is attributed to the wind velocity. The ambiguity of the issue of the wind velocity is a strong motivation to revisit the interpretation of SN 1997eg observations and reassess CSM parameters. It should be emphasised that the wind velocity is crucial for the problem of the SN IIn origin. The velocity of $10-30 \mathrm{~km} \mathrm{~s}^{-1}$ would indicate that a presupernova before the explosion was a red supergiant (RSG), whereas the wind velocity of $\sim 200 \mathrm{~km} \mathrm{~s}^{-1}$ would imply that presupernova could be a luminous blue variable (LBV). The latter are actually associated with some SNe IIn, e.g., SN 2009ip (Pastorello et al. 2013).

The primary goal of the paper is the determination of the presupernova wind velocity and density. The presentation starts with the description and realization of the model for the emission line profiles of $\mathrm{H} \alpha$ and $\mathrm{He}$ I $5876 \AA$. As a result we recover the velocity of the undisturbed supernova envelope at the reverse shock; this velocity will be used then for the determination of the wind density in the SN/CSM interaction model. To recover the wind velocity I propose the model for the narrow $\mathrm{H} \alpha$ formed in the the slow wind with the preshock acceleration by the cosmic ray (CR) precursor. In addition, the wind velocity and gas temperature will be recovered in the region of the coronal [FeX] $6374 \AA$ line emission. The SN/CSM interaction then will be modelled to derive the wind density. In the last section I discuss results and make suggestions on the nature of the presupernova.

Following Hoffman et al. (2002) the SN 1997eg distance of $40 \mathrm{Mpc}$ is adopted. The explosion moment is rather uncertain. However high expansion velocity and their fast decrease indicate that supernova was detected soon after the explosion. Therefore in accord with 
Hoffman et al. (2002) we adopt that the supernova exploded 9 days before the discovery.

\section{Broad emission lines and supernova expansion velocity}

The supernova expansion in a dense CSM, created by the presupernova wind, brings about forward and reverse shocks. Between them a cool $\left(T \sim 10^{4} K\right)$ dense shell forms, since the reverse shock is strongly radiative; outer shock can be partially radiative or adiabatic depending on the wind density. The supernova deceleration manifested in the decrease of the width of broad line wings can be used to recover the wind density. The appropriate decceleration indicator is the maximum velocity of the undisturbed supernova envelope $\left(v_{s n}\right)$ at the reverse shock that can be estimated from the maximum velocity in the blue wing of the $\mathrm{H} \alpha$ emission line, since the red wing is affected by the Thomson scattering. Yet the velocity jump between supernova and dense shell $\left(v_{s n}>v_{d s}\right)$ can result in the blue wing enhancement due to Thomson scattering and the photon blueshift into the radial velocities $<-v_{s n}$ (an analogue of the Fermi acceleration). Therefore, a reliable method for the supernova velocity determination should be based on the modelling of the profiles of broad emission lines.

A general model for the formation of emission lines in SNe IIn is lacking though such a model is unlikely plausible given highly heterogeneous SNe IIn family. Yet a source of the emission is undoubtedly the dissipation of the kinetic energy in shocks. As already mentioned, strong emission lines, particularly, H $\alpha$ in the SN 1997eg spectrum are composed by three components: broad, narrow, and intermediate (Hoffman et al. 2008). The narrow component originated in the CSM is irrelevant to the issue of supernova expansion velocity, so it will be addressed in a separate section. However, it should be noted that at the early stage the narrow line emitted by the CSM may acquire broad wings formed due to the multiple Thomson scattering of the line photons in the optically thick circumstellar cocoon as occured in early SN 1998S (Chugai 2001). For SN 1997eg, however this effect is negligible at the considered late stages.

The schematic picture of the supernova interaction with the CSM showing zones for the emission of the broad, narrow, and intermediate components is presented in Fig. 1. It should be mentioned that in fact the broad component consists of two componens: the emission of the undisturbed supernova envelope and of the dense shell residing between two shocks. The modelling of the line profile from the undisturbed supernova ejecta with the kinematics of $v=r / t$ and adopted distribution of the emissivity versus radius is a rather simple problem. More complicated model however is needed to describe the broad line originated from the dense shell, the issue addressed below. 


\subsection{Emission line from dense shell}

In one-dimensional approach the CDS is very thin in accord with the momentum flux conservation $\Delta R / R \sim\left(v_{t h} / v_{d s}\right)^{2} \sim 10^{-6}-10^{-5}$ (where $v_{t h} \sim 10 \mathrm{~km} \mathrm{~s}^{-1}$ is the thermal velocity of the CDS). There are two problems with the line emission from that thin shell. The radiation of the optically thick line, e.g. H $\alpha$, escapes the thin CDS in the surface regime. The line width in the comoving frame for the optical depth $<10^{5}$ is $\Delta \nu \lesssim 6 v_{t h} / \lambda$. Even for the thermalized line source function the expected $\mathrm{H} \alpha$ luminosity from the CDS $L_{32}=4 \pi^{2} R_{d s}^{2} B_{\nu}(T) \Delta \nu$ is relatively low. Another problem is that the optically thick line in that case has an M-shaped profile (Gerasimovič 1933), which is not the case for SN 1997eg.

In reality, the CDS is liable to the strong deformation due to the Rayleigh-Taylor instability (Chevalier \& Blondin 1995, Blondin \& Ellison 2001), which results in the penetration of spikes of dense cool gas into the hot gas of the forward shock wave followed by mixing and formation of a complicated three-dimensional structure of the cool dense gas in the form of corrugated two-dimensional elements. This structure with a low volume filling factor resides in a spherical layer of the width of $\Delta R / R \sim 0.1$ (Blondin \& Ellison 2001). The contact surface area $S$ of the cool dense gas in this case significantly exceeds the initial surface area $4 \pi R^{2}$, so the area ratio $A=S /\left(4 \pi R^{2}\right) \gg 1$. The formation of the optically thick line emitted by the cool dense gas in this case was considered earlier and the main result is that for the area ratio $A \gg 1$ the line luminosity gets larger, while the line profile is significantly modified compared to the case of spherical thin shell (Chugai et al. 2004). Hereafter when talking about the line from the CDS we mean the line from the strongly deformed CDS.

In the analytic model (Chugai et al. 2004) the distribution of the CDS material is described by an ensemble of random thin disks. Each disk is considered as a particle that can emit and absorb resonance radiation. Transport of the radiation in this shell is treated in the Sobolev approximation. The probability for the photon escape in the direction defined by cosine $\mu$ of the angle between wave vector and the radius is

$$
\beta=\left(1-\mathrm{e}^{-Q}\right) / Q, \quad Q=Q_{0} /\left(1-\mu^{2}\right)
$$

A zero velocity radial gradient is adopted, which is a good approximation. The parameter $Q_{0}$ depends on area ratio $A$, thermal width of the local line absorption coefficient, relative width of the mixing layer $\Delta R / R$ and the line optical depth of a single disk. The propability $\beta$ actually is a macroscopic analogue of the Sobolev escape probability. The effect of the $Q_{0}$ value on the optically thick line is as follows: for $0.1<Q_{0}<0.5$ the profile is flat-topped, while for $Q_{0} \gtrsim 1$ the profile becomes parabolic. 


\subsection{Intermediate component}

The intermediate component, that contributes significantly to $\mathrm{H} \alpha$, originates from the interaction of the forward shock with dense CS clouds (Chugai \& Danziger 1994). The characteristic cloud shock speed $v_{c}$ is determined by the density contrast of the cloud relative to the intercloud gas $\chi=\rho_{c} / \rho_{i c}$ and the forward postshock velocity that is comparable to the velocity of the CDS $v_{d s}$ :

$$
v_{c} \approx 2 v_{d s} / \sqrt{\chi}
$$

where the prefactor of 2 takes account of the four times larger postshock density. For $\chi \sim 10^{2}$ and $v_{d s}=5000 \mathrm{~km} \mathrm{~s}^{-1}$ the expected cloud shock speed is $v_{c} \sim 10^{3} \mathrm{~km} \mathrm{~s}^{-1}$, a typical value for the intermediate component. Higher velocities in the wings of the intermediate component are the outcome of the acceleration of cloud fragments in the post-forward shock flow. The maximal velocities of cloud fragments turn out to be comparable to the postshock velocity of forward shock (Klein et al. 1994). One should bear in mind that the cloud shock is essentially radiative, so cloud fragments are rather dense and cool $\left(T \sim 10^{4} \mathrm{~K}\right)$.

The modelling of the intermediate component requires setting the emissivity distribution versus velocity and radius, $j(v, r)$. I consider the homogeneous source distribution along the radius and the parametric form for $j(v)$ on the interval $v_{\min }<v<v_{\max }$, with $v_{\min } \sim v_{c}$ and $v_{\max } \lesssim v_{d s}$ :

$$
j(v) \propto\left\{\begin{array}{cc}
\frac{v-v_{\min }}{v_{c}-v_{\min }}, & \text { if } v_{\min }<v<v_{c}, \\
{\left[\frac{v_{\max }-v}{v_{\max }-v_{c}}\right]^{q},} & \text { if } v_{c}<v<v_{\max }, \\
0, & \text { otherwise. }
\end{array}\right.
$$

The power index $q$ in this expression is close to unity. The function $j(v)$ has a maximum at the typical velocity of the cloud shock $v=v_{c}$ that is a free parameter. The range $v_{c, \text { min }}<v<v_{c}$ where $j(v)$ grows is introduced to take into account the scatter of clouds properties. Without this detail the profile would be flat-topped which is not observed. We adopt $v_{\text {min }}=0.9 v_{c}$. The radial distribution is set to be uniform in the range $\delta R \sim 0.2 R$. Somewhat similar form of $j(v, r)$ was used earlier (Chugai 2009) for the line profiles in SN 2006jc.

\subsection{H $\mathrm{H}$, HeI $5876 \AA$ and velocity of supernova envelope}

The model for the broad lines includes three line-emitting zones: (1) homologously expanding supernova envelope $\left(v=r / t, r<R_{0}=1\right)$, (2) the disturbed CDS $\left(R_{0}<r<\right.$ $R_{1}=1.1$ ) with the average velocity $v_{d s}$ and random velocities in the range $\pm 0.05 v_{d s}$ (Blondin 
\& Elison 2004), and (3) the cool dense gas of fragmented CS clouds in the forward shock $\left(R_{0}<r<R_{2}=1.2\right)$. First two zones are responsible for the broad component, while the third one for the intermediate component. We neglect the thickness of the reverse shock and adopt the radius of the undisturbed supernova envelope to be equal $R_{0}$. The relative contribution of the supernova envelope into the line emission is $f_{s n}$, the contribution of the CDS is $f_{d s}$, and the rest fraction $1-f_{d s}-f_{s n}$ is contributed by the intermediate component.

The Monte Carlo technique is employed for the radiation transfer computation. The model profiles of $\mathrm{H} \alpha$ and He I $5876 \AA$ are compared to the observed spectra for three epochs, 57, 80, and 204 days after the discovery in Figures 2, 3, and 4. Observed spectra are taken from the database Weizmann supernova data repository (Yaron \& Gal-Yam 2012). Model parameters are presented in Table, which contains starting from the first column: the moment after the discovery, velocity of the undisturbed supernova envelope, CDS velocity, maximal velocity of the gas responsible for the intermediate component, power index in the expression (3), parameter $Q_{0}$ in equation (1), optical depth of the undisturbed supernova and the CDS in continua, single scattering albedo in ejecta and the CDS, contribution of ejecta and CDS to $\mathrm{H} \alpha$ and He I $5876 \AA$. The adopted CS cloud shock speed $v_{c}$ is $850 \mathrm{~km} \mathrm{~s}^{-1}$ on days 57 and 80, and $920 \mathrm{kms}^{-1}$ on day 204.

On days 57 and 80 the broad component is the same for the He I $5876 \AA$ and $\mathrm{H} \alpha$ lines; in both cases the homogeneous source distribution in ejecta is adopted. On day 204 for He I $5876 \AA$ the line emissivity in supernova is concentrated towards rhe center $j \propto 1 / v$, whereas for $\mathrm{H} \alpha$ as before $j=$ const. Remarkably, the intermediate component of He I $5876 \AA$ is significantly weaker than in $\mathrm{H} \alpha$. This probably reflects a low excitation degree in the dense CS cloud fragments responsible for the intermediate component. On day 204 the components are not easily distiguished visually, so in this case profiles of all the components are shown.

The primary goal of the line profile modelling is the velocity of the undisturbed supernova at the reverse shock (Table, second column). The relative uncertainty of this value is $10 \%$. It is instructive to compare these values to the visual measurements of the maximal velocity in the $\mathrm{H} \alpha$ blue wing. On days 57, 80, and 204 thus estimated values are equal to $10000 \mathrm{~km} \mathrm{~s}^{-1}, 8500 \mathrm{~km} \mathrm{~s}^{-1}$, and $7000 \mathrm{~km} \mathrm{~s}^{-1}$, respectively, in agreement with values in Table. The uncertainty of both the CDS velocity and $v_{\max }$ is $15 \%$. Other parameters are determined with the uncertainty of $20 \%$ and these parameters fortunately do not affect inferred supernova velocities. 


\section{Narrow lines and wind velocity}

The high resolution spectrum of SN $1997 \mathrm{eg}\left(7 \mathrm{~km} \mathrm{~s}^{-1}\right)$ on day 198 (Salamanka et al. 2002) shows along with narrow emission lines (FWHM $30-40 \mathrm{~km} \mathrm{~s}^{-1}$ ) also the $\mathrm{H} \alpha$ absorption component with the radial velocity in the blue wing of $-160 \mathrm{~km} \mathrm{~s}^{-1}$. It is natural to account for that high velocity in the same way as suggested for the broad absorption component of narrow $\mathrm{H} \alpha$ of SN 1998S (type IIL/n) (Chugai et al. 2002). The central point of the interpretation of the absorption profile is that the high velocity is attributed to the preshock gas accelerated by the CR precursor that is a feature of the diffusive shock acceleration mechanism (Druri \& Fölk 1981).

The CR pressure at the shock $\left(p_{c}\right)$ required to provide the preshock gas acceleration up to $u=160 \mathrm{~km} \mathrm{~s}^{-1}$ can be estimated from the equation of the momentum flux conservation. The integration in the preshock region $-\infty<x<0$ gives:

$$
\left(p+\rho v^{2}\right)_{0}-\left(p+\rho v^{2}\right)_{-\infty}=0 .
$$

Neglecting the gas density variation in the preshock and using sensible approximations $v_{\infty} \approx$ $v_{d s}, p_{-\infty}=0, p_{0}=p_{c}, v_{0}=v_{d s}-u$, and making use of $u / v_{d s} \ll 1$ one gets from equation (4) $p_{c} / \rho v_{f s}^{2}=2\left(u / v_{f s}\right)$. For $u=160 \mathrm{~km} \mathrm{~s}^{-1}$ and $v_{f s}=5000 \mathrm{~km} \mathrm{~s}^{-1}$ we infer the ratio of the CR pressure to the upstream gas dynamic pressure to be $p_{c} / \rho v_{f s}^{2} \approx 0.06$. This is rather modest requirement given claims that up to $50 \%$ of the postshock pressure in supernova remnants may be due to the CR (Helder et al. 2009).

Preempting the $\mathrm{H} \alpha$ modelling it is instructive to derive velocity of the undisturbed wind using the strongest coronal line of $[\mathrm{FeX}] 6374 \AA$ in the same spectrum. In Figure 5 the observed [FeX] 6374 $\AA$ line (Salamanka et al. 2002) is shown with the overplotted model. For stationary wind the best fit is found for the wind speed of $19.7 \mathrm{~km} \mathrm{~s}^{-1}$ and the Doppler broadening for $T=0.9 \cdot 10^{6} \mathrm{~K}$. The accuracy of the derived values is $10 \%$. The inferred temperature is close to the value of $1.1 \cdot 10^{6} \mathrm{~K}$ that corresponds to the maximum of the relative abundance of Fe X ion (Burgess \& Seaton 1964) in a coronal approximation.

The kinematic model we use for the $\mathrm{H} \alpha$ suggests that the wind velocity decreses from $160 \mathrm{~km} \mathrm{~s}^{-1}$ to $20 \mathrm{~km} \mathrm{~s}^{-1}$ in the radius range of $1.2<r<1.4$ and remains constant futher on. The region of the $\mathrm{H} \alpha$ formation is bounded by the radius $1.6 R_{0}$, because anyway the population of the second level decreases with the radius due to the Ly $\alpha$ escape. In the optimal model the second level population smoothly drops by a factor of 1.7 in the range $1.2<r<1.6$. We adopt a thermal Doppler broadening for the local absorption coefficient $k=k_{0} \exp \left(-\left[\Delta \lambda / \Delta \lambda_{D}\right]^{2}\right)$, where $\Delta \lambda$ is the wavelenth displacement from the central wavelength, and $\Delta \lambda_{D}=\lambda_{0}\left(v_{t h} / c\right)$ is the Doppler width, $v_{t h}=\left(2 k_{B} T N_{A}\right)^{1 / 2}, k_{B}$ is the Boltzmann constant, $T$ is the gas temperature, $N_{A}$ is the Avogadro number. The scattering in the line 
frequences is assumed to be conservative with the complete frequency redistribution. As a background radiation for narrow line we consider emission in the broad and intermediate components of the $\mathrm{H} \alpha$. In the ejecta and CDS we adopt the same extinction as in the model on day 204. The optimal model (Fig. 5) reproduces the observed narrow $\mathrm{H} \alpha$ for the wind optical depth in $\mathrm{H} \alpha$ of 0.4 , wind velocity of $20 \mathrm{~km} \mathrm{~s}^{-1}$, and thermal velocity of hydrogen of $30 \mathrm{~km} \mathrm{~s}^{-1}$. The latter corresponds to the gas temperature of $5.4 \cdot 10^{4} \mathrm{~K}$.

The major result of this modelling is that the $\mathrm{H} \alpha$ narrow emission component forms primarily in the wind with the velocity of $20 \mathrm{~km} \mathrm{~s}^{-1}$, which supports the result of the [Fe X] $6374 \AA$ modelling. In this regard a question arises on the difference between the temperature derived from $\mathrm{H} \alpha\left(5 \cdot 10^{5} \mathrm{~K}\right)$ and from $[\mathrm{FeX}] 6374 \AA\left(9 \cdot 10^{5} \mathrm{~K}\right)$. A possible explanation is that in the inner wind, where the $\mathrm{H} \alpha$ forms, the temperature is indeed lower than in the outer wind, where the coronal lines form. The reason could be a thermal instability, when a sharp transition between cold and hot states is plausible for similar specific heating rates similar to the sharp transition between chromosphere and corona.

\section{Supernova deceleration and wind density}

The interaction of the supernova with the CSM is modelled in the thin shell approximation (Chevalier 1982, Guiliani 1982). I recap only essential points, since the model has been described earlier (e.g. Chugai et al. 2004). The gas swept up by the forward and reverse shock forms the shell that is considered to be thin $(\delta r / r \ll 1)$. Its motion is governed by the equation of motion and equation of the mass conservation. The optical bolometric luminosity is adopted to be equal to the luminosity of both shocks. The shock luminosity at the moment $t$ is calculated as the shock kinetic luminosity multiplied by the radiation efficiency $\eta=t /\left(t+t_{c}\right)$, where $t_{c}$ is the cooling time of the postshock gas for the density four times of the preshock density. We omit the radiation diffusion effect and the luminosity powered by the internal energy of the supernova explosion. The density distribution in the supernova ejecta is set as $\rho=\rho_{0} /\left[1+\left(v / v_{0}\right)^{8}\right]$, where $\rho_{0}$ and $v_{0}$ are specified by the ejecta mass $M$ and kinetic energy $E$. The adopted density distribution is very close to that of hydrodynamic models of a SNe IIP.

The high expansion velocity of the SN $1997 \mathrm{eg}$ envelope on day $57\left(\approx 10^{4} \mathrm{~km} \mathrm{~s}^{-1}\right)$ indicates a very weak decceleration of outer layers. This in turn suggests that in the inner region the density is significantly lower than that extrapolated inward according to the steady wind $\left(\rho \propto r^{-2}\right)$. Indeed, our simulations show the law $\rho \propto r^{-2}$ in the region $r<4 \cdot 10^{13} \mathrm{~cm}$ does not permit to describe both the luminosity and the velocity evolution. The successful model (Fig. 6) suggests the CSM with the homogeneous density for $r<r_{b}=4 \cdot 10^{15}$ and 
$\rho \propto r^{-2}$ for $r>r_{b}$. The shown estimate of the bolometric luminosity is obtained using photometry by Tsvetkov \& Pavlyuk (2004). The adopted supernova ejecta mass in this model is $15 M_{\odot}$. With this choice the optimal kinetic energy is $2.2 \cdot 10^{51} \mathrm{erg}$. The recovered wind density parameter in the range $r \geq r_{b}$ is $w=\dot{M} / u=5 \cdot 10^{16} \mathrm{~g} \mathrm{~cm}^{-1}$. With the found wind density and the wind velocity of $20 \mathrm{~km} \mathrm{~s}^{-1}$ the presupernova mass loss rate turns out to be $1.6 \cdot 10^{-3} M_{\odot} \mathrm{yr}^{-1}$. It should be emphasised that the found wind density is insensitive to the adopted ejecta mass. E.g., the model with $M=1 M_{\odot}$ and $E=4.5 \cdot 10^{50}$ erg reproduces observation as good as the $15 M_{\odot}$ model for the same wind density. The reason for the model degeneracy with respect to the mass is related to the power law distribution of supernova density $\rho(v)$ in outer layers that can be the same for the different appropriate values of $M$ and $E$.

\section{Discussion}

The wind density parameter and the wind velocity $\left(20 \mathrm{~km} \mathrm{~s}^{-1}\right)$ are the major results of the new interpretation of the available observational data on SN 1997eg. These parameters imply the presupernova mass loss rate of $1.6 \cdot 10^{-3} M_{\odot} \mathrm{yr}^{-1}$. The CDS radius on day 213 is $1.26 \cdot 10^{16} \mathrm{~cm}$. The wind material with the velocity of $20 \mathrm{~km} \mathrm{~s}^{-1}$ at this radius was lost 200 years before the explosion. Remarkably, the new estimate of the wind velocity is significantly (8 times) lower than the previusly adopted value of $160 \mathrm{~km} \mathrm{~s}^{-1}$.

The central point of our interpretation of narrow lines in the spectrum of SN1997eg is the conjecture that high velocities detected in the absorption blue wing of narrow $\mathrm{H} \alpha$ are due to the wind acceleration in the CR precursor of the shock wave. This suggestion is in line with the earlier interpretation of a similar phenomenon in the $\mathrm{H} \alpha$ absorption of SN 1998S (Chugai et al. 2002). Thus at present we have two cases of SN IIn that demonstrate in the $\mathrm{H} \alpha$ a signature of the preshock wind acceleration by the CR precursor. There is a suspicion that in the high resolution spectrum of SN 2002ic (SN IIn) on day 265 (Kotak et al. 2004) we see a similar phenomenon: the $\mathrm{H} \alpha$ narrow emission has a width FWHM of $80 \mathrm{~km} \mathrm{~s}^{-1}$, whereas the absorption blue wing extends to $-250 \mathrm{~km} \mathrm{~s}^{-1}$. The preshock acceleration in the CR precursor is observed in the Tycho supernova remnant as well. Lee et al. (2007) detected gas velocities in the preshock region in the range of $60-130 \mathrm{~km} \mathrm{~s}^{-1}$ that have been attributed to the gas acceleration in the $\mathrm{CR}$ precursor. This range of velocities corresponds to the ratio 0.03 - 0.06 with respect to the shock velocity. With this ratio extended to the SN 1997eg one expects to find preshock velocities of $150-300 \mathrm{~km} \mathrm{~s}^{-1}$ in line with the observed value of $160 \mathrm{~km} \mathrm{~s}^{-1}$.

Let us turn now to implications of the inferred CR pressure for the the radio emission 
from SN 1997eg. The radio flux from SN 1997eg at $3.6 \mathrm{~cm}$ on days 177 and 186 was detected at the level of $0.5 \mathrm{mJy}$ (Lassey \& Weiler 1998). This flux corresponds to the radio luminosity $\nu L_{\nu}=8.4 \cdot 10^{36} \mathrm{erg} \mathrm{s}^{-1}$. The intercloud wind with the coronal temperature of $10^{6} \mathrm{~K}$ and the shock radius of $R=1.24 \cdot 10^{16} \mathrm{~cm}$ suggests the opticall depth to the $f f$-absorption of 1.45 , so the unabsorbed radio luminosity should be of $3 \cdot 10^{37} \mathrm{erg} \mathrm{s}^{-1}$.

We assume that the width of the radio-emitting shell is $\Delta R=0.1 R$ and it is filled with relativistic electrons with the energy density $\epsilon_{e}=3 K_{e p} p_{c}=1.26 \cdot 10^{-2} \mathrm{erg} \mathrm{cm}^{-3}$, where we adopt the standard electron-to-proton ratio $K_{e p}=10^{-2}$, the found ratio $p_{c} / \rho v_{f s}^{2}=0.06$, with $v_{f s}=5200 \mathrm{~km} \mathrm{~s}^{-1}$ and inferred wind density. The Lorentz factor of radio-emitting electrons at $3.6 \mathrm{~cm}$ is $\gamma=55 B^{-0.5}$. For the standard energy spectrum of relativistic electrons $d n / d E \propto$ $E^{-2}$ in the range $E_{1}<E<E_{2}$ the radio luminosity is $\nu L_{\nu}=\epsilon_{e} V /\left(2 t_{s} \ln \left(E_{2} / E_{1}\right)\right)$, where $V$ is the volume of the radio-emitting shell, $t_{s}=3 m_{e} c^{2} /\left(4 \sigma_{T} c W_{m} \gamma\right)$ is the characteristic time of synchrotron losses, and $W_{m}=B^{2} / 8 \pi$. For sensible energy range of accelerated electrons $E_{1}=1 \mathrm{MeV}, E_{2}=10^{3} \mathrm{MeV}$ one gets $\nu L_{\nu}=1.3 \cdot 10^{38} B^{3 / 2} \mathrm{erg} \mathrm{s}^{-1}$. The magnetic field required to account for the unabsorbed radio luminosity is thus $B \approx 0.4$ G. Remarkably, this value is in the range of $0.1-0.6 \mathrm{G}$ inferred for 12 radio supernovae through synchrotron self-absorption effects (Chevalier (1998). The seemingly large value of the magnetic field of SN 1997eg thus turns out quite reasonable.

It is instructive to compare the model and observational $\mathrm{H} \alpha$ luminosities in order to verify the consistency of the broad line modelling. On day 204 the model suggests that the Thomson optical depth of the unshocked ejecta is $\tau_{T}=0.6$. For the CDS radius at this age of $r=1.26 \cdot 10^{16} \mathrm{~cm}$ one gets the average electron number density $n_{e}=7.2 \cdot 10^{7} \mathrm{~cm}^{-3}$. Assuming the solar composition and the $\mathrm{H} \alpha$ effective recombination coefficient $\alpha_{32}=3.4 \cdot 10^{-13} \mathrm{~cm}^{3}$ $\mathrm{c}^{-1}$ for $T_{e}=5000 \mathrm{~K}$ (Osterbrock 1989) one obtains the $\mathrm{H} \alpha$ recombination luminosity of the unshocked ejecta $L_{s n}=4.4 \cdot 10^{40} \mathrm{erg} \mathrm{s}^{-1}$. Given the contribution of unshocked ejecta to the overall $\mathrm{H} \alpha$ of 0.27 we come to the total $\mathrm{H} \alpha$ luminosity in our model of $1.6 \cdot 10^{41} \mathrm{erg} \mathrm{s}^{-1}$ that coinsides with the observed $\mathrm{H} \alpha$ luminosity of $1.7 \cdot 10^{41} \mathrm{erg} \mathrm{s}^{-1}$ (Salamanca et al. 2002).

To which extent the inferred wind density is consistent with other observational data? One can find the model luminosity of the coronal [Fe X] $6374 \AA$ line on day 198 and compare it to the observed luminosity of $1.5 \cdot 10^{38} \mathrm{erg} \mathrm{s}^{-1}$ (Salamanka et al. 2002) for the distance of $40 \mathrm{Mpc}$. The maximum fraction of FeX ion to iron $\left(f_{10}=0.33\right)$ is attained for the temperature of $\approx 10^{6} \mathrm{~K}$ (Burgess \& Seaton 1964). The collisional excitation coefficient for that temperature is $3.8 \cdot 10^{-9} \mathrm{~cm}^{3} \mathrm{~s}^{-1}$ (Aggarval \& Keenan 2005). The emission measure in the region $r>1.45 \cdot 10^{16} \mathrm{~cm}$ for the wind density parameter $w=1.6 \cdot 10^{-3} M_{\odot} \mathrm{yr}^{-1}$ assuming the solar composition reproduces the observed [FeX] $6374 \AA$ luminosity, if the mass fraction of the hot intercloud wind is $f_{i c}=0.6$. The latter is realistic value and the agreement 
between the model and observations thus turns out acceptable.

Another estimate of the wind density can be obtained from the ratio of nebular to auroral lines of O III. Taking into account errors of the determination of observed fluxes of [O III] lines in the noisy spectrom on day 198 (Salamanca et al. 2002, Figure 3) the ratio $F(5007+4959) / F(4363)$ turns out to be in the range $0.5-1$. For the temperature of $10^{5} \mathrm{~K}$ corresponding to the maximum fraction of the O III ion (Böhringer 1998) the found ratio suggests the electron number density of $(0.75-1.7) \cdot 10^{7} \mathrm{~cm}^{-3}$ (Osterbrock 1989). This range is in accord with the preshock electron number density $n_{e}=1.4 \cdot 10^{7} \mathrm{~cm}^{-3}$ implied by the inferred wind density.

The recovered wind velocity $\left(20 \mathrm{~km} \mathrm{~s}^{-1}\right)$ implies that the presupernova of SN 1997eg was RSG, not LBV supergiant as earlier suggested on the basis of the wind velocity of $160 \mathrm{~km} \mathrm{~s}^{-1}$ (Salamanka et al. 2002, Hoffman et al. 2008). Some SNe IIn indeed are associated with LBV presupernovae, e.g., SN 2009ip, in which case the wind velocity is of $240 \mathrm{~km} \mathrm{~s}^{-1}$ (Pastorello et al. 2013), and the luminous presupernova is identified with the massive LBV $M_{m s} \gtrsim 60 M_{\odot}$ (Foley et al. 2011). The insight into the initial mass of SN 1997 eg is provided by the sample of 255 RSG in M31 (Massey \& Evans 2016). This sample lies on the HR-diagram below the evolutionary track for $37 M_{\odot}$, which implies the SN 1997 eg progenitor mass $<40 M_{\odot}$. The mass lower limit should be around $25 M_{\odot}$ since according to a general wizdom stars from the mass range of $9-25 M_{\odot}$ explode as SNe IIP (Woosley et al. 2002). It is remarkable that the relation between the mass loss rate and RSG luminosity, viz., $\dot{M}=10^{-4}\left(L / 10^{5} L_{\odot}\right)^{2.1}$ (Salasnich et al. 1999) combined with the mass loss rate of $1.6 \cdot 10^{-3} M_{\odot} \mathrm{yr}^{-1}$ suggests the SN 1997eg progenitor of $30 M_{\odot}$, in accord with the estimated range of $25-40 M_{\odot}$.

\section{Conclusion}

The interpretation and modelling of the available spectral and photometric data on type IIn supernova SN 1997eg permits us to infer major parameters of the presupernova mass loss during the last $200 \mathrm{yr}$ before the explosion: the wind velocity of $20 \mathrm{~km} \mathrm{~s}^{-1}$ and mass loss rate

of $1.6 \cdot 10^{-3} M_{\odot} \mathrm{yr}^{-1}$. The recovered wind density is consistent with the value independently estimated from the flux of the coronal line [Fe X] 6374 . The wind properties indicate that the presupernova at the final evolutionary stage was a red supergiant with the large mass loss rate. The progenitor mass at the main sequence propably was in the range of $25-40 M_{\odot}$. 


\section{REFERENCES}

Aggarwal K. M., Keenan F. P., Astron. Astrophys. 431, 1215 (2005)

Böhringer H., Lecture Notes in Physics 506, 341 (1998)

Blondin J. M., Ellison D. C., Astrophys. J. 560, 244 (2001)

Burgess A., Seaton M. J., Mon. Not. R. Astron. Soc. 127, 355 (1994)

Woosley S. E., Blinnikov S. I., Heger A., Nature 450, 390 (2007)

Woosley S. E., Heger A., Weaver T. A., Rev. Mod. Phys. 74, 1015 (2002)

Gerasimovič B. P., Zeitschrift für Astrophysik 7, 335 (1933)

Giuliani J. L., Astrophys. J. 245, 903 (1981)

Drury L. O’C., Völk J. H., Astrophys. J. 248, 344 (1981)

Klein R. I. , McKee C. F., Colella P., Astrophys. J. 420, 213 (1994)

Kotak R., Meikle W. P. S, Adamson A., Leggett S. K., Mon. Not. R. Astron. Soc. 354, L13 (2004)

Lee J.- J., Koo B.- C., Raymond J. et al., Astrophys. J. 659, 133 (2007)

Lacey C. K., Weiler K. W., Sramek R. A., van Dyk C. D., IAUC, 7068 (1998)

Massey P., Evans K. A., Astrophys. J. 826, 224 (2016)

Nakano S., Aoki M., IAU Circ. 6790 (1997)

Osterbrock D. E., Astrophysics of Gaseous Nebulae and Active Galactic Nuclei (Mill Valley, California, University Science Books, 1989), p.19

Pastorello A., Cappellaro E., Inserra C. et al., Astrophys. J. 767, 1 (2013)

Salamanca I., Terlevich R. J., Tenorio-Tagle G., Mon. Not. R. Astron. Soc. 330, 844 (2002)

Salasnich B., Bressan A., Chiosi C., Astron. Astrophys. 342, 131 (1999)

Smith N., McCray R., Astrophys. J. 671, 17 (2007)

Stathakis R. A., Sadler E. M., Mon. Not. R. Astron. Soc. 250, 786 (1991)

Filippenko A. V., Barth A. J., IAUC 6794, 1 (1997) 
Filippenko A. V., Supernovae (Springer Verlag, New York, 1991), p.467

Helder E. A., Vink J., Bassa C. G., et al., Science 325, 719 (2009)

Hoffman J., Leonard D. C., Chornock R., Filippenko A. V., Barth A. J., Matheson T., Astrophys. J. 688, 118 (2008)

Tsvetkov D. Yu., Pavlyuk N. N., Aston. Lett. 30, 32

Foley R. J., Berger E., Fox O. et al., Astrophys. J. 732, 32 (2011)

Chugai N. N., Mon. Not. R. Astron. Soc. 400, 866 (2009)

Chugai N. N., Chevalier R. A., Lundqvist P., Mon. Not. R. Astron. Soc. 355, 627 (2004)

Chugai N. N., Blinnikov S. I., Fassia A., Lundqvist, P., Meikle W. P. S., Sorokina E. I., Mon. Not. R. Astron. Soc. 330, 473 (2002)

Chugai N. N., Danziger I. J., Mon. Not. R. Astron. Soc. 268, 173 (1994)

Chevalier R. A., Astrophys. J. 499, 810 (1998)

Chevalier R. A., Blondin J. M., Astrophys. J. 444, 312 (1995)

Chevalier R. A., Astrophys. J. 259, 302 (1982)

Yaron O., Gal-Yam A., Publ. Astron. Soc. Pacific 124, 668 (2012) 
Table 1: Parameters of models for the $\mathrm{H} \alpha$ and He I $5876 \AA$ lines

\begin{tabular}{l|c|c|c|c|c|c|c|c|c|c|c|c|c}
\hline Days & $v_{s n}$ & $v_{d s}$ & $v_{\max }$ & $q$ & $Q_{0}$ & $\tau_{s n}$ & $\tau_{d s}$ & $\omega_{s n}$ & $\omega_{d s}$ & $f_{s n}$ & $f_{d s}$ & $f_{s n, H e}$ & $f_{d s, H e}$ \\
\hline 57 & 9500 & 7000 & 5000 & 1.5 & 0.5 & 0.9 & 0.13 & 0.4 & 0.4 & 0.5 & 0.1 & 0.66 & 0.3 \\
80 & 8500 & 6100 & 4500 & 1.1 & 0.7 & 0.7 & 0.1 & 0.4 & 0.4 & 0.42 & 0.19 & 0.7 & 0.25 \\
204 & 7100 & 5200 & 4300 & 1 & 1 & 0.6 & 0.05 & 1 & 0.3 & 0.27 & 0.33 & 0.68 & 0.3 \\
\hline
\end{tabular}




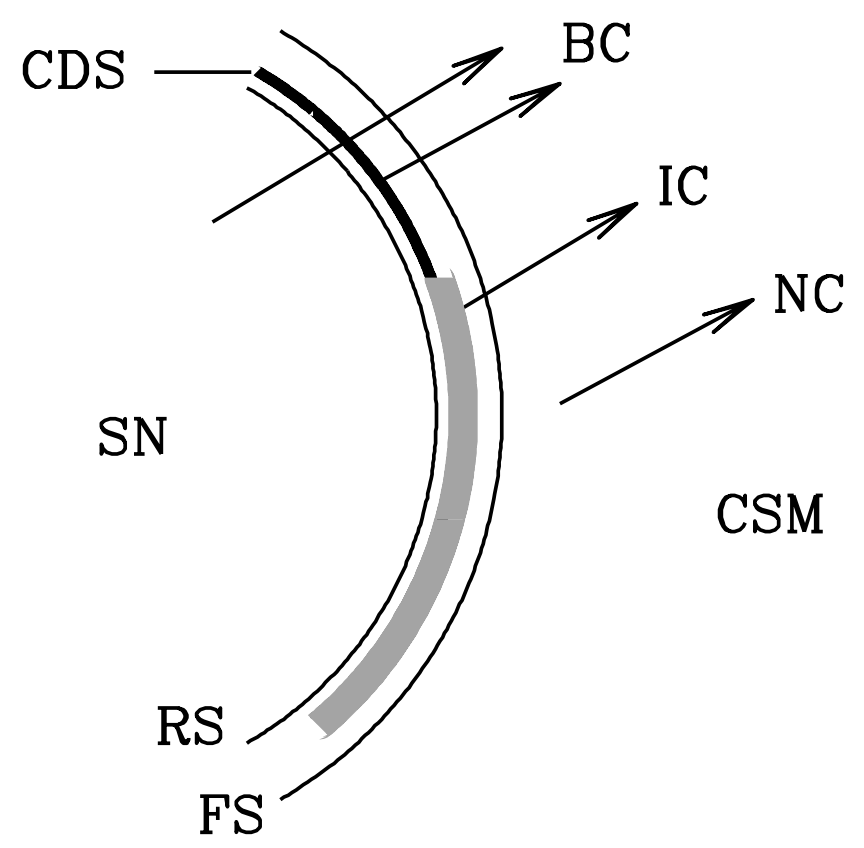

Fig. 1.- Schematic picture of the supernova (SN) interaction with the circumstellar matter (CSM), that shows regions of the formation of three components of an emission line: narrow component (NC), broad component (BC), and intermediate component (IC). Two thin arc lines correspond to the forward shock (FS) and reverse shock (RS); thick line shows the cool dense shell (CDS); grey arc shows a zone which contains cool dense gas of shocked and fragmented circumstellar clouds; this layer is responsible for the intermediate component. 

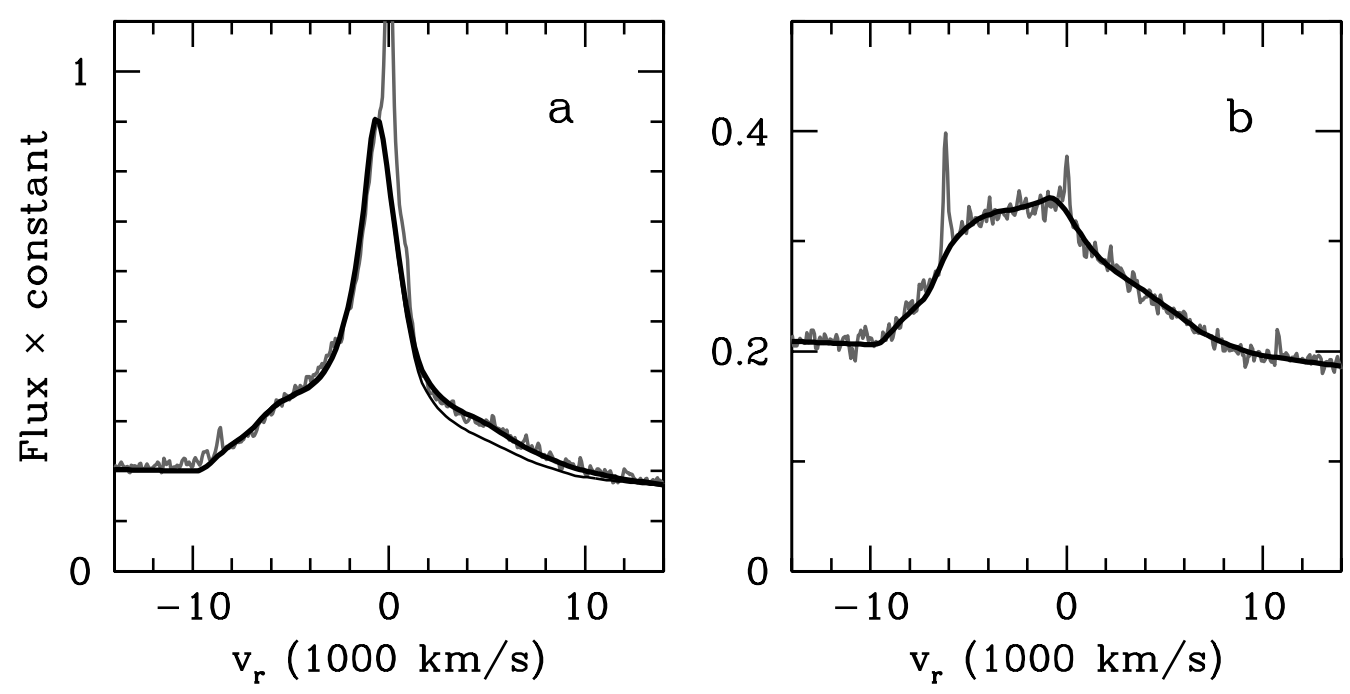

Fig. 2.- $\mathrm{H} \alpha$ (panel a) and HeI $5876 \AA$ (panel b) lines in the SN 1997 eg spectrum on day 57 compared to models (cf. Table). Thin line on the panel a shows the model without the contribution of HeI $6674 \AA$. 

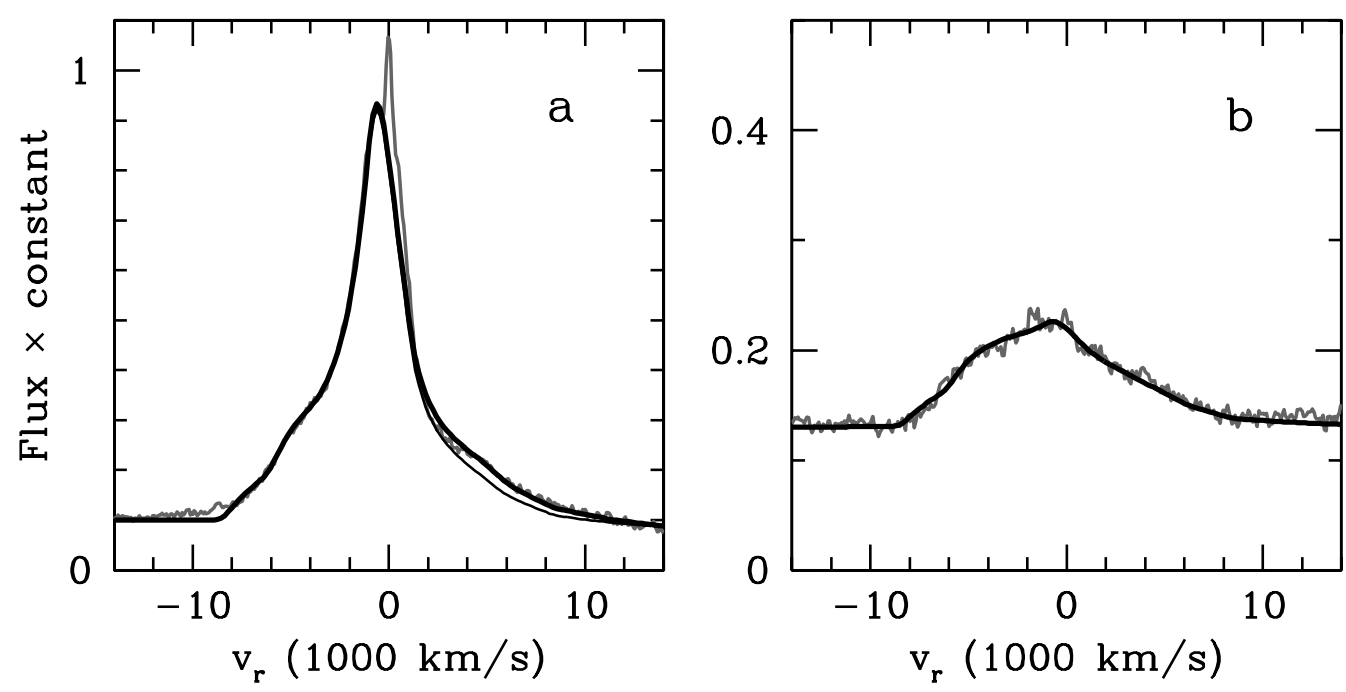

Fig. 3.- The same as Fig. 2 but for day 80 . 

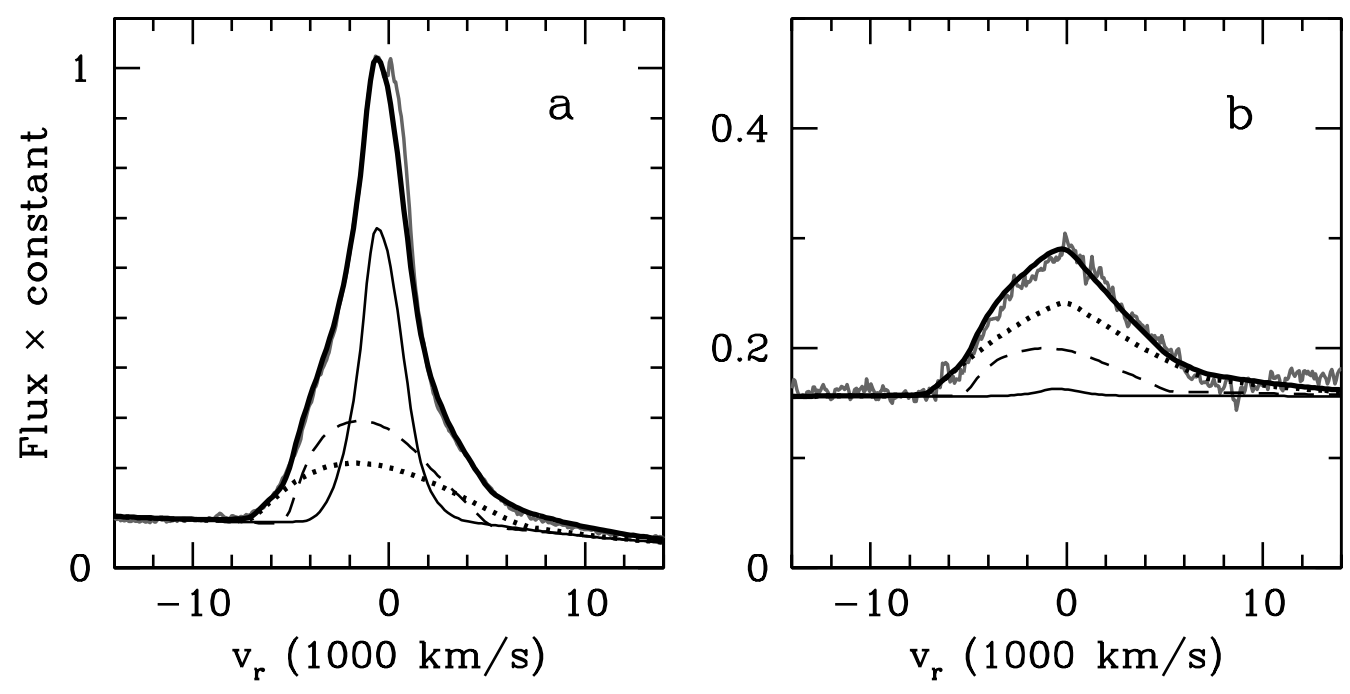

Fig. 4.- The same as Fig. 2 but for day 204. Additionally shown are the intermediate component (thin solid line), the contribution of the dense shell (dashed line), and the contribution of undisturbed supernova ejecta (dotted). 

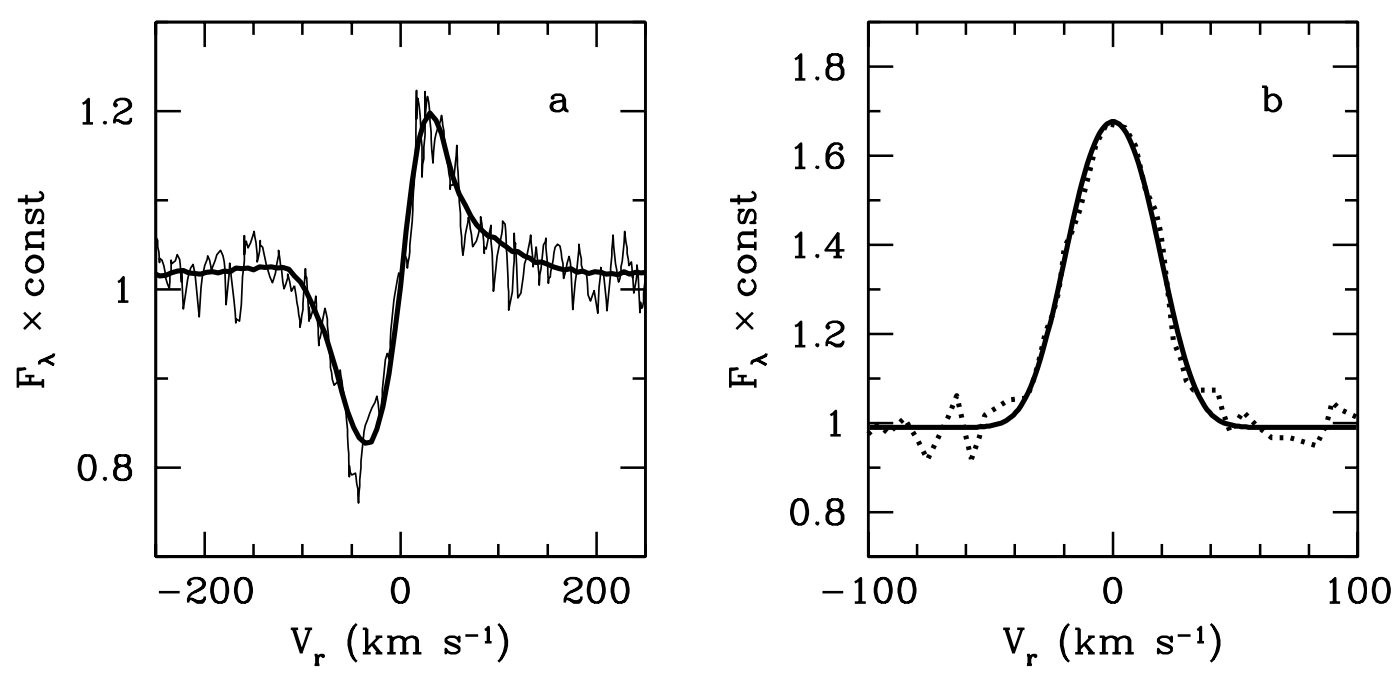

Fig. 5.- Circumstellar $\mathrm{H} \alpha(\mathbf{a})$ and $[\mathrm{FeX}] 6374 \AA$ (dotted) in the SN 1997eg spectrum on day 198 compared to models (thick lines). 

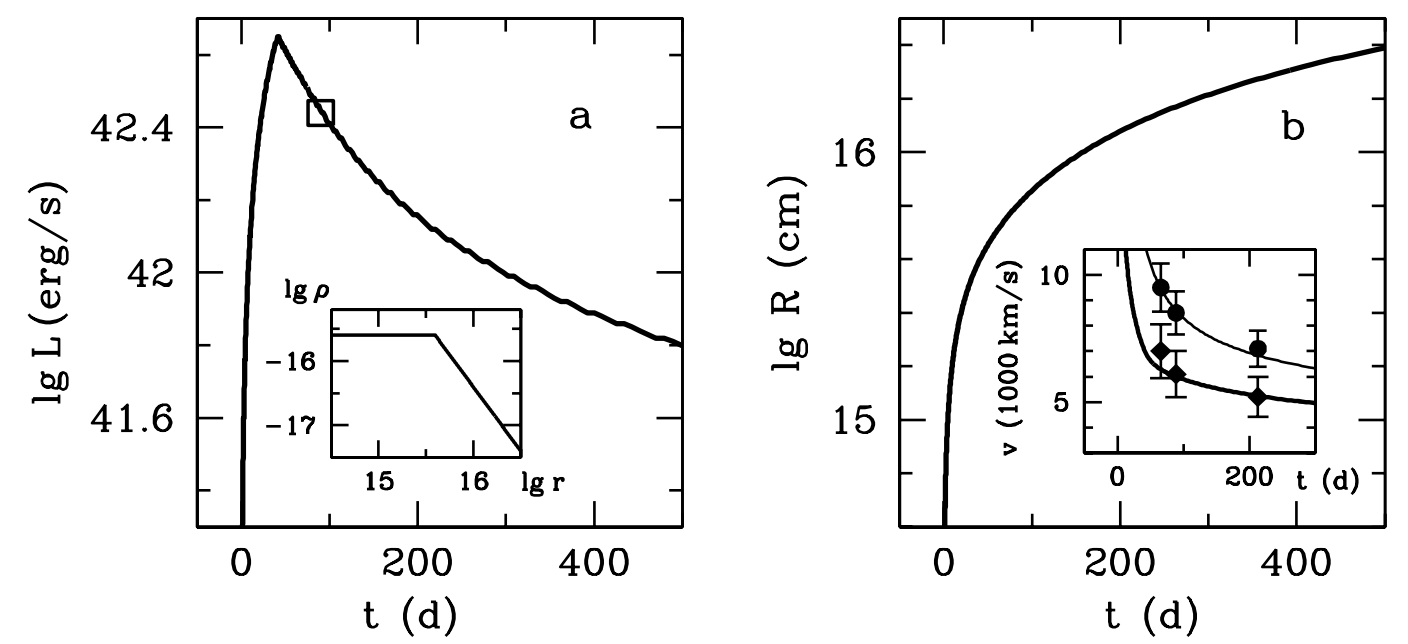

Fig. 6.- Bolometric light curve (panel a) and radius of the dense shell (panel b) in the model of $15 M_{\odot}$ supernova ejecta interaction with the CSM. In the panel a the square is the bolometric luminosity according to photometry data (Tsvetkov \& Pavlyuk 2004). The inset in panel a shows the density distribution of the CSM in the model. The inset in panel $\mathbf{b}$ shows the model supernova velocity at the reverse shock (thin line) compared to the velocity estimates from the $\mathrm{H} \alpha$ (filled circles) and the model velocity of the dense shell compared to estimates from the $\mathrm{H} \alpha$ (diamonds). 